\title{
Nephrology Referral in Diabetes Practice
}

\author{
Sanjay Kalra ${ }^{1}$ Manisha Sahay ${ }^{2}$ Rakesh Sahay ${ }^{3}$ \\ ${ }^{1}$ Department of Endocrinology, Bharti Hospital, Karnal, \\ Haryana, India \\ 2Department of Nephrology, Osmania Medical College, Hyderabad, \\ Telangana, India \\ ${ }^{3}$ Department of Endocrinology, Osmania Medical College, \\ Hyderabad, Telangana, India
}

\begin{abstract}
Address for correspondence Sanjay Kalra, MD, DM, Department of Endocrinology, Bharti Hospital, Karnal 132001, Haryana, India (e-mail: brideknl@gmail.com).
\end{abstract}

J Soc Health Diab 2018;6:106-108.

\author{
Abstract \\ Keywords \\ - chronic kidney disease \\ - diabetic kidney \\ disease \\ - diabetic nephropathy \\ - end-stage renal \\ disease \\ - type 1 diabetes \\ - type 2 diabetes
}

Diabetic nephropathy, including diabetic kidney disease (DKD), is a major complication of diabetes. Appropriate management of DKD requires multispecialty intervention under the guidance of endocrinology and nephrology. However, delayed referral to specialized nephrology services usually occurs, and this leads to suboptimal outcomes. This multispecialty consensus suggests simple clinical and biochemical parameters that should prompt referral of DKD patients to nephrology. A checklist for physicians who manage DKD is also provided.

\section{Introduction}

Kidney disease is common in diabetes. ${ }^{1}$ In spite of great advances in diagnosis and management of diabetic kidney disease (DKD), renal outcomes still remain suboptimal. One of the main reasons for this is delayed referral to nephrology specialist clinics. Such delay may occur due to multiple causes, including nonavailability of qualified professionals. Often, however, this is because of lack of awareness.

\section{Barriers to Referral}

Many diabetes care providers may not be aware that all kidney disease in persons with diabetes is not DKD. Some authors use DKD as a broad term, which has two varieties: diabetic nephropathy (DN) and non-DKD. Other authors use DKD interchangeably with DN. ${ }^{2,3}$ It may also be difficult to gauge the severity, achieve therapeutic targets, or manage multiple comorbid conditions that need attention in kidney disease.

\section{Need for Referral}

Data show that multidisciplinary endocrinology and nephrology care in the DKD clinic is associated with a lower risk of end-stage renal disease (ESRD) and improves outcomes. ${ }^{4}$ Timely care of the kidney also helps in prevention of progression of chronic kidney disease. ${ }^{5}$ A nephrology referral, therefore, helps in tertiary prevention of diabetes and secondary prevention of diabetes complications. Appropriate nephrology intervention in DKD reduces the risk of cardiovascular complications as well.

\section{Indications for Referral}

However, all persons with diabetes cannot be referred to nephrology care. The number of qualified nephrologists is inadequate to take care of all persons with DKD, let alone diabetes.

The Indian Council of Medical Research (ICMR) 2018 guidelines on type 2 diabetes mellitus state that "if there are high risk factors in the patient and/or there is any abnormality in history, examination and preliminary investigations, these patients need to be referred to specialists for further management." ${ }^{\prime 6}$

The ICMR calls for referral of DN patients to a nephrologist if these is severe/resistant hypertension, serum potassium greater than $5.5 \mathrm{mEq} / \mathrm{L}$, nephritic range proteinuria, proteinuria in the absence of retinopathy, serum creatinine greater than $1.5 \mathrm{mg} \%$, pregestational diabetes or gestational diabetes mellitus with proteinuria, estimated glomerular filtration 
rate (eGFR) less than $60 \mathrm{~mL} / \mathrm{min}$ (as calculated by Modification of Diet in Renal Disease [MDRD]), or fluid overload/oliguria. While these eight points are a comprehensive coverage of the various complications of DN, they are presented in a manner that may complicate the subject for the unexperienced physician.

The Research Society for Study of Diabetes in India (2017) guidelines for management of type 2 diabetes ${ }^{7}$ lay down four indications for referral of DN to a nephrologist: eGFR less than $30 \mathrm{~mL} / \mathrm{min} / 1.73 \mathrm{~m}^{2}$, progressive deterioration of kidney function, persistent proteinuria, biochemical or fluid retention, or difficulty in diagnosis (to rule out nondiabetic renal disease). These indications, though easy to follow, may not help all deserving patients access nephrology services in a timely manner.

\section{Suggested Best Practice}

Hence, a simple yet comprehensive algorithm is required to triage persons who need specialist referral. A list of such indications is mentioned in - Table 1. It must be noted that referral may vary depending on the ability and interest of the primary care physician/diabetologist/endocrinologist in

Table 1 Indications for nephrology referral in diabetes

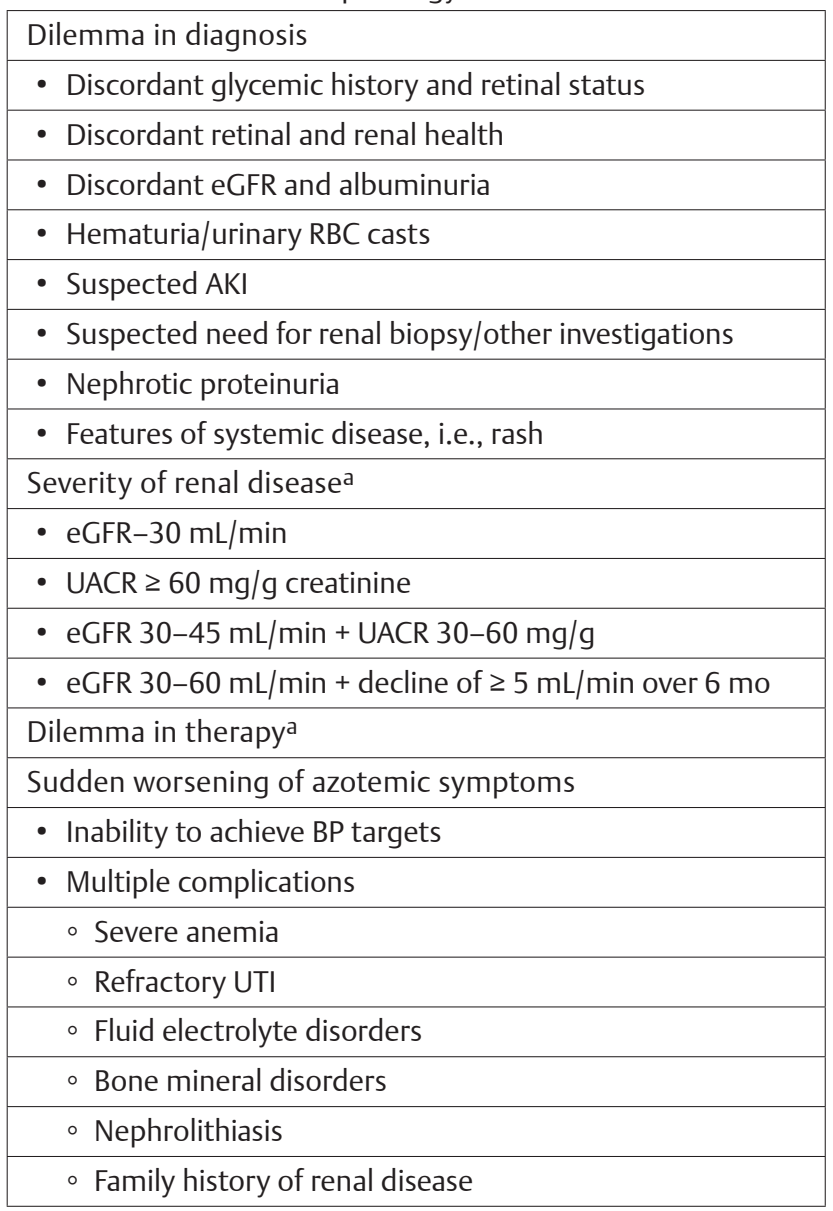

Abbreviations: AKI, acute kidney injury; BP, blood pressure; eGFR, estimated glomerular filtration rate; RBC, red blood cells; UACR, urinary albumin creatinine ratio; UTI, urinary tract infection.

aAll values must be confirmed by repeat testing.
Table 2 Physician's responsibility in nephrology care for persons with diabetes

\begin{tabular}{|l|}
\hline All persons with diabetes \\
\hline Check UACR yearly along with serum creatinine and eGFR \\
\hline All persons with eGFR $30-60 \mathrm{~mL} / \mathrm{min}$ \\
\hline $\begin{array}{l}\text { Rubri-health: Hemoglobin, transferrin saturation, serum } \\
\text { iron, and ferritin }\end{array}$ \\
\hline $\begin{array}{l}\text { Bone disease: Serum calcium, phosphorus, alkaline } \\
\text { phosphatase, parathormone, vitamin D }\end{array}$ \\
\hline Vaccination: Hepatitis B and pneumococcal vaccine \\
\hline Institution of statins for diabetics with CKD > 18 y \\
\hline Stop smoking/tobacco \\
\hline $\begin{array}{l}\text { Education: Sick day rules, diet, avoidance of nephrotoxic } \\
\text { drugs, judicious use of radiocontrast, }\end{array}$ \\
\hline Preserve hand veins \\
\hline All persons with GFR < $30 \mathrm{~mL} /$ min \\
\hline Refer to nephrology service \\
\hline
\end{tabular}

Abbreviations: CKD, chronic kidney disease; eGFR, estimated glomerular filtration rate; GFR, glomerular filtration rate; UACR, urinary albumin creatinine ratio.

treating DKD. A physician who is interested and well trained in nephrology may not feel the need to refer stable DKD patients. Conversely, referral may be increased as such a physician will be able to suspect non-DKD, such as glomerulonephritis or renal vasculitis, more frequently. A basic checklist for all physicians is mentioned in - Table 2 .

\section{The Nephrologist's Role}

Most people with DKD reach the nephrology after ESRD has occurred, and complications have set on. Timely referred allows the nephrologist to institute preventive and therapeutic measures, designed to retard the progression of renal impairment, minimize complications, and enhance quality of life. Regular monitoring may cause acute kidney injury and worsen renal function. Therapeutic counseling, performed in an empathic and knowledgeable manner, allows the patient and her family to prepare for life ESRD, using dialysis and renal transplant as treatment options.

\section{Summary}

While these suggestions are not absolute, they do provide a framework upon which to build a pragmatic referral policy. Such a policy will be of relevance to the majority of health care systems operating in South Asia and beyond.

\section{References}

1 Saran R, Robinson B, Abbott KC, et al. US Renal Data System 2016 annual data report: epidemiology of kidney disease in the United States. Am J Kidney Dis 2017;69(3, Suppl 1):A7-A8

2 Thomas MC, Cooper ME, Zimmet P. Changing epidemiology of type 2 diabetes mellitus and associated chronic kidney disease. Nat Rev Nephrol 2016;12(2):73-81 
3 Tuttle KR, Bakris GL, Bilous RW, et al. Diabetic kidney disease: a report from an ADA Consensus Conference. Am J Kidney Dis 2014;64(4):510-533

4 Low S, Lim SC, Wang J, et al. Long term outcomes of patients with type 2 diabetes attending a multidisciplinary diabetes kidney disease clinic. J Diabetes 2017

5 American Diabetes Association. 10. Microvascular Complications and Foot Care: Standards of Medical Care in Diabetes-2018. Diabetes Care 2018;41(Suppl 1):S105-S118
6 The Indian Council of Medical Research (ICMR). Guidelines for Management of Type 2 Diabetes. New Delhi, India: ICMR; 2018

7 Bajaj S. RSSDI clinical practice recommendations for the management of type 2 diabetes mellitus 2017. Int J Diabetes Dev Ctries 2018;38(1, Suppl 1):1-115 